\title{
Anaemia in pregnancy in the district of Anuradhapura, Sri Lanka - need for updating prevalence data and screening strategies
}

\author{
U Chathurani', I Dharshika', D Galgamuwa', N D Wickramasinghe' ${ }^{1}$, T C Agampodi', S B Agampodi ${ }^{1}$ \\ (Index words: anaemia in pregnancy, prevalence, screening)
}

\begin{abstract}
Objectives To determine the prevalence of anaemia during pregnancy in Anuradhapura district and evaluate present screening methods for anaemia.

Methods Modified WHO 30 cluster sampling method with increased precision was used to estimate the prevalence of anaemia in the Anuradhapura district, Sri Lanka. Serum haemoglobin was measured using methemoglobin method. Clinical examination was carried out to evaluate the conjunctival method in anaemia screening. Values recorded from haemoglobin colour scale method used in the field antenatal clinics were collected.
\end{abstract}

Results A total of 990 pregnant women participated in the study. In the first, second and third trimesters, prevalence of anaemia was $7.6 \%, 19.7 \%$ and $19.3 \%$ respectively. Gestational age adjusted anaemia prevalence among pregnant women in this study population was $14.1 \%$ (95\% Cl 12.0-16.4\%). Mean and median haemoglobin concentration of the study sample was $11.8 \mathrm{~g} / \mathrm{dL}$ (SD $1.02 \mathrm{~g} / \mathrm{dl}$ and IQR $11.2-12.5 \mathrm{~g} / \mathrm{dl}$ ). Among anaemic pregnant women, average values for Mean Corpuscular Volume (MCV), Mean Corpuscular Haemoglobin ( $\mathrm{MCH}$ ), and Mean Corpuscular Haemoglobin Concentration (MCHC) were, $82.9 \mathrm{fl}$ (SD 11.5), 27.6 (SD-3.6) pg/cell and $32.9 \mathrm{~g} / \mathrm{dl}$ (SD 1.8 ) respectively. Sensitivity and specificity of haemoglobin colour scale method was $50 \%$ (95\% Cl 29.0-71.0\%) and $76.3 \%(95 \% \mathrm{Cl} 66.9-83.7 \%)$ respectively. Sensitivity and specificity of conjunctival method in detecting anaemia during pregnancy was $18.8 \%(95 \% \mathrm{Cl} 11.9-28.4)$ and $69.3 \%(95 \% \mathrm{Cl} 58.2-$ $78.6 \%)$.

Conclusions Prevalence of anaemia in the district of Anuradhapura was less than $50 \%$ of the estimated prevalence for Sri Lanka. Both haemoglobin colour scale and conjunctival method were having low validity in detecting anaemia in pregnancy.

Ceylon Medical Journal 2012; 57: 101-106

\section{Introduction}

Anaemia in pregnancy has been a long known major maternal morbidity condition in Sri Lanka. Most recent WHO estimates show that the prevalence of anaemia during pregnancy in Sri Lanka as 29.3\% [1]. Sri Lanka Demographic and Health Survey (DHS) data in 2007 shows similar results with an overall anaemia prevalence of $34 \%$, with $20.7 \%$ mild anaemia and $13.3 \%$ moderate to severe anaemia [2]. Sri Lankan maternal health programme contain several evidence based strategies to prevent anaemia during pregnancy. Iron folate supplementation, malaria chemoprophylaxis, routine antihelminthic treatment, regular clinical examination and screening for anaemia using $\mathrm{Hb}$ colour scale (HCS) method. Though routine serum $\mathrm{Hb}$ measurements in the booking visit and around 34 weeks are also recommended, lack of laboratory facilities and resources impede universal application of latter recommendation in Sri Lanka.

National and international estimates of anaemia in pregnancy are not consistent with several recent studies. A study done in the Puttalam district in 1999 reported a prevalence of $14.4 \%$ and another study conducted in the adjacent Gampaha district in 2001-2002 period reported $7.1 \%$ prevalence [3]. In 2009 , a $8.2 \%$ prevalence was reported from the Vavuniya district [5]. The prevalence was $18-23.4 \%$ in Colombo. Though these sample survey results show a wide range, all these show a lower prevalence than the national estimates of DHS and WHO. Routinely reported data from the maternal health management information system provides the lowest estimates. In Anuradhapura, these routine data shows that the prevalence of anaemia was only $0.19 \%$ (for 19,240 registered pregnancies) in 2009.

To develop local strategies and to prioritise public health interventions, sub national data on public health problems are essential. Inconsistent data on anaemia prevalence could be due to actual sub national variations, problems of survey designs, or poor quality data in routine surveillance. The present study was conducted to provide proper estimates of anaemia prevalence in Anuradhapura, and to determine the major socio-demographic factors associated with anaemia in this area. We also evaluated

${ }^{1}$ Department of Community Medicine, Faculty of Medicine and Allied Sciences, Rajarata University of Sri Lanka.

Correspondence: SBA, e-mail: <sunethagampodi@yahoo.com>. Received 27 April and revised version accepted 26 May 2012. Competing interests: none declared. 
present screening strategies for anaemia in pregnancy in Sri Lanka.

\section{Methods}

Present study was conducted in the Anuradhapura district. Total estimated population residing in Anuradhapura is 830,000 , of which, $92.7 \%$ are rural [6]. In Anuradhapura, more than 19,000 pregnant mothers are registered annually at antenatal clinics. In 2009, 15,417 deliveries were reported through the public health information system and $98.1 \%$ of these births were reported as institutional deliveries [7].

Modified WHO 30 cluster sampling technique with increased precision was used to estimate the prevalence of anaemia in the Anuradhapura district [8]. Study was conducted in two phases. The first phase was as a part of a larger morbidity assessment study conducted in the Anuradhapura district and the second phase was exclusively for anaemia in pregnancy, in which additional information were collected on probable determinants of anaemia during pregnancy and further investigations were also carried out. We previously published the first phase of the study and the second phase of the study is outlined here [9].

The study population for the present study included all pregnant women registered in the Anuradhapura district field $\mathrm{MOH}$ clinics. Reported pregnant mother registration in field clinics was $100 \%$ in the Anuradhapura district, indicating that the clinic based probability sampling technique would represent a representative sample of all pregnant women from the area [7]. In the first stage of sampling, five $\mathrm{MOH}$ areas were selected randomly from the Anuradhapura district. In the second stage, field clinics were selected to place 30 clusters using probability proportionate to size. To achieve the calculated sample size, from each cluster, 17 pregnant women were required. However, at the clinic, all pregnant women were invited to participate in the study after explaining the study objectives and procedures. This was done to minimise ethical issues, because most of the pregnant women were only having the $\mathrm{Hb}$ colour scale method results, which was shown to be having low reliability [10].

The main outcome variable for the prevalence study was the anaemia status based on serum haemoglobin levels. We used WHO classification of anaemia for pregnant women aged 15-49 years; normal ->10.9 g/dl; mild anaemia - 10.0-10.9 g/dl; moderate anaemia - 7.0-9.9 $\mathrm{g} / \mathrm{dl}$; severe anaemia $-<7.0 \mathrm{~g} / \mathrm{dl}[11]$.

An interviewer administered questionnaire was used to collect data on socio-demographic, and pregnancy related factors. Results of previous $\mathrm{Hb}$ tests (if available) were obtained from the antenatal record. A clinical examination was conducted to evaluate the validity of routine clinical method of anaemia evaluation. Since the data included culturally sensitive questions on menstruation, only female data collectors were used in this study. A venous blood sample was obtained from all pregnant mothers by a trained nurse. Blood samples were sent to the reference laboratory and the $\mathrm{Hb}$ assessment was carried out using cyanmethaemoglobin method on the same day of sample collection.

Assuming that the prevalence of anaemia is $30 \%$ and with a cluster effect of 1.5, 484 pregnant mothers were required for the sample to achieve 5\% precision and $95 \%$ confidence limits. Number of clusters were predesigned as 30 , thus the size of the cluster was 17 and the minimum sample size needed for the prevalence study was 510 pregnant women.

All quantitave variables in this study were analysed as categorical variables. Income and menstrual bleeding data were collected as continuous variables, but analysed as quartiles. Prevalence of anaemia was analysed as proportions and $95 \%$ confidence limits. Comparison of continuous variables was achieved using student's t test. Categorical variables were tested using the chi-square or Fisher's exact test. Gestational age adjusted anaemia prevalence was calculated using direct standardisation method.

Approval for this study was obtained under the ethical clearance for maternal mortality project from the Research and Ethics Committee, Rajarata University of Sri Lanka. Written informed consent was obtained from the study participants. $\mathrm{Hb}$ results were sent to the pregnant women through respective $\mathrm{MOH}$. Women who were having moderate to severe anaemia were referred to tertiary care centres.

\section{Results}

Total number of pregnant women who participated in the prevalence study was 990. Mean age of the study sample was 27.0 years (SD 5.5 years) and $9.0 \%$ of the study sample was teenagers.

The mean haemoglobin concentration of the study sample was $11.8 \mathrm{~g} / \mathrm{dL}$ (SD $1.02 \mathrm{~g} / \mathrm{dL}$ ). Distribution of $\mathrm{Hb}$ in the study population was slightly skewed to left (Figure $1)$. Based on the WHO criteria, only a single pregnant woman $(0.1 \%)$ had severe anaemia, which had a $\mathrm{Hb}$ concentration of $6.7 \mathrm{~g} / \mathrm{dL}$. She was 30 years old with a gestational age of 20 weeks and was in her second pregnancy. At 8 weeks of POA her BMI was 17.9. Her first pregnancy was uneventful and she was not having any history of diagnoses of chronic diseases, anaemia or menstrual disorders. Red cell indices showed microcytic (MCV- 70 fl) hypochromic (MCH 22.4 pg/cell) anaemia.

In first, second and third trimesters, prevalence of anaemia was $7.6 \%, 19.7 \%$ and $19.3 \%$ respectively (Table 1 and Figure 2). Gestational age adjusted anaemia prevalence in this study population was $14.1 \%(95 \%$ CI $12.0-16.4 \%)$. Observed difference of anaemia prevalence between first trimester verses second and third trimester was statistically significant (Chi square 8.77, $\mathrm{p}=0.013$ ). Mean $\mathrm{Hb}$ levels in first second and third trimester was 12.4 (SD .96), 11.6 (SD .99) and 11.7 (SD 1.0) g/dl. 
Table 1. Prevalence of mild, moderate and severe anaemia by trimester

\begin{tabular}{|c|c|c|c|c|c|c|c|c|c|c|}
\hline & \multicolumn{10}{|c|}{ Anaemia prevalence } \\
\hline & \multirow[t]{2}{*}{$\begin{array}{c}\text { Mean } \\
g / d L\end{array}$} & \multirow[t]{2}{*}{$H b(S D)$} & \multicolumn{2}{|c|}{$\begin{array}{l}\text { Severe } \\
(<7 g / d l)\end{array}$} & \multicolumn{2}{|c|}{$\begin{array}{l}\text { Moderate } \\
(7-9.9 \mathrm{~g} / \mathrm{dl})\end{array}$} & \multicolumn{2}{|c|}{$\begin{array}{c}\text { Mild } \\
(10-10.9 \mathrm{~g} / \mathrm{dl})\end{array}$} & \multicolumn{2}{|c|}{ Total } \\
\hline & & & $n$ & $\%$ & $n$ & $\%$ & $n$ & $\%$ & $n$ & $\%$ \\
\hline First trimester & 12.38 & $(.96)$ & 0 & .0 & 0 & .0 & 10 & 7.6 & 10 & 7.6 \\
\hline Second trimester & 11.63 & $(.99)$ & 1 & .4 & 11 & 4.6 & 35 & 14.7 & 47 & 19.7 \\
\hline Third trimester & 11.74 & $(1.0)$ & 0 & .0 & 23 & 3.7 & 97 & 15.6 & 120 & 19.3 \\
\hline Total & 11.78 & $(1.02)$ & 1 & .1 & 34 & 3.4 & 142 & 14.3 & 177 & 19.3 \\
\hline
\end{tabular}

Table 2. Distribution of mean hemoglobin levels by socio-demographic characteristics

\begin{tabular}{|c|c|c|c|}
\hline & Mean & $S D$ & ANOVA \\
\hline \multicolumn{4}{|l|}{ Age category } \\
\hline$>20$ years & 11.69 & .96 & $\mathrm{~F}=.747$ \\
\hline 20-34 years & 11.80 & 1.03 & $\mathrm{P}=.474$ \\
\hline$>35$ years & 11.87 & .98 & \\
\hline \multicolumn{4}{|l|}{ Highest level of education } \\
\hline Up to grade 5 & 11.77 & 1.17 & $\mathrm{~F}=1.943$ \\
\hline Up to grade 10 & 11.78 & 1.00 & $\mathrm{P}=.121$ \\
\hline Passed O/L & 11.71 & 1.10 & \\
\hline Passed A/L & 11.93 & .94 & \\
\hline \multicolumn{4}{|l|}{ Main economic activity } \\
\hline Professionals/lesser professionals & 12.19 & .93 & $\mathrm{~F}=1.828$ \\
\hline Clerical service and skilled & 11.81 & .95 & $\mathrm{P}=.14$ \\
\hline Unskilled manual workers & 11.56 & 1.04 & \\
\hline Housewife & 11.79 & 1.03 & \\
\hline \multicolumn{4}{|c|}{ Total monthly income (quartiles) } \\
\hline Poorest & 11.77 & 1.10 & $\mathrm{~F}=.409$ \\
\hline 2 nd & 11.86 & 1.04 & $\mathrm{P}=.746$ \\
\hline $3 \mathrm{rd}$ & 11.79 & .94 & \\
\hline Richest & 11.78 & .95 & \\
\hline \multicolumn{4}{|l|}{ Parity } \\
\hline First & 11.84 & .99 & $\mathrm{~F}=.996$ \\
\hline Second & 11.82 & 1.08 & $\mathrm{P}=.394$ \\
\hline Third & 11.73 & 1.02 & \\
\hline Fourth or more & 11.68 & .91 & \\
\hline \multicolumn{4}{|l|}{ BMI (before 12 weeks of POA) } \\
\hline$<18$ & 11.76 & 1.07 & $\mathrm{~F}=3.331$ \\
\hline $18-22.9$ & 11.76 & 1.06 & $\mathrm{P}=.036^{*}$ \\
\hline$>22.9$ & 11.96 & .93 & \\
\hline
\end{tabular}

* Significant at 0.05 level, BMI - Body Mass Index, SD - Standard Deviation, ANOVA - Analysis of Variance, POA - period of amenorrhea

Table 3. Comparison of conjunctival method and cynoheamoglobin method in assessing anaemia during pregnancy

\begin{tabular}{lccccc}
\hline & \multicolumn{2}{c}{$\mathrm{Hb}<11 \mathrm{~g} / \mathrm{dl}$} & \multicolumn{2}{c}{$\mathrm{Hb} \geq 11 \mathrm{~g} / \mathrm{dl}$} \\
& $\mathrm{n}$ & 69 & 81.2 & 398 & 88.4 \\
Not pale & 15 & 17.6 & 46 & 10.2 \\
Mild pallor & 1 & 1.2 & 6 & 1.3 \\
\hline Moderate pallor & 15 & & \multicolumn{2}{c}{$\%$} \\
\hline
\end{tabular}

*no severe pallor or plethoric pregnant women were detected among this study sample 


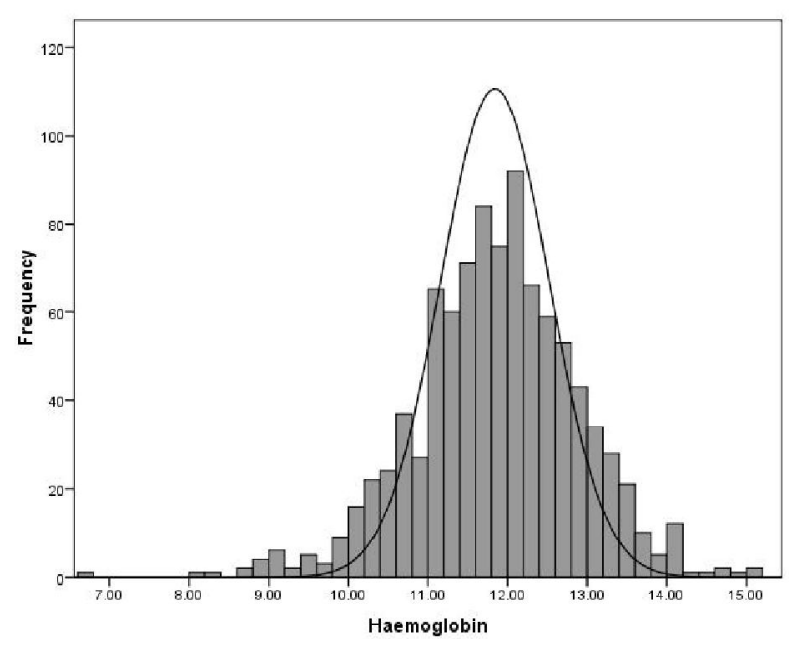

Figure 1. Distribution of serum haemoglobin concentration.

Serum haemoglobin levels were compared across socio-demographic groups to identify factors associated with anaemia (Table 2). Mean $\mathrm{Hb}$ concentration of pregnant women with BMI more than or equal to $23.0 \mathrm{~kg} /$ $\mathrm{m} 2$ was 11.96 (SD .93), compared to $11.76 \mathrm{~g} / \mathrm{dl}$ among those with BMI less than $23.0 \mathrm{~kg} / \mathrm{m}^{2}$. This difference was statistically significant $(\mathrm{p}=.036)$

Red cell indices were available for 66 mothers who were detected as having anaemia during the second phase of the study. Average values for Mean Corpuscular Volume (MCV), Mean Corpuscular Haemoglobin (MCH), and Mean Corpuscular Haemoglobin Concentration among anaemic mothers were, $82.9 \mathrm{fl}$ (SD 11.5), 27.6 (SD3.6) $\mathrm{pg} /$ cell and 32.9g/dl (SD 1.8) respectively. Prevalence of microcytic hypochromic anaemia was $3.4 \%$ and normocytic normochromic anaemia was $10.5 \%$. No macrocytic anaemia was detected in this study sample.

Validity of HCS and conjunctival method were evaluated in the second phase of the study. HCS values were available for $115(21.3 \%)$ pregnant women. These values were recorded in pregnancy records as ranked ordered data, and usually done by PHM or public health nursing sisters during antenatal clinics.

Correlation between HCS and $\mathrm{Hb}$ values showed statistically significant but weak correlation (Spearmen's rho .262, p .005). However, HCS method detected only 9 out of 18 anaemic women with 23 false positives. Sensitivity and specificity of HCS method was 50\% (95\% CI $29.0-71.0 \%)$ and $76.3 \%$ (95\% CI $66.9-83.7 \%)$ respectively.

Clinical examination of conjunctivae yielded 61 (11.4\%) pregnant women with mild pallor and 7 (1.3\%) with moderate pallor (Table 3 ). Sensitivity and specificity of conjunctival method in detecting anaemia during pregnancy was $18.8 \%(95 \% \mathrm{CI} 11.9-28.4)$ and $69.3 \%(95 \%$ CI 58.2-78.6\%).

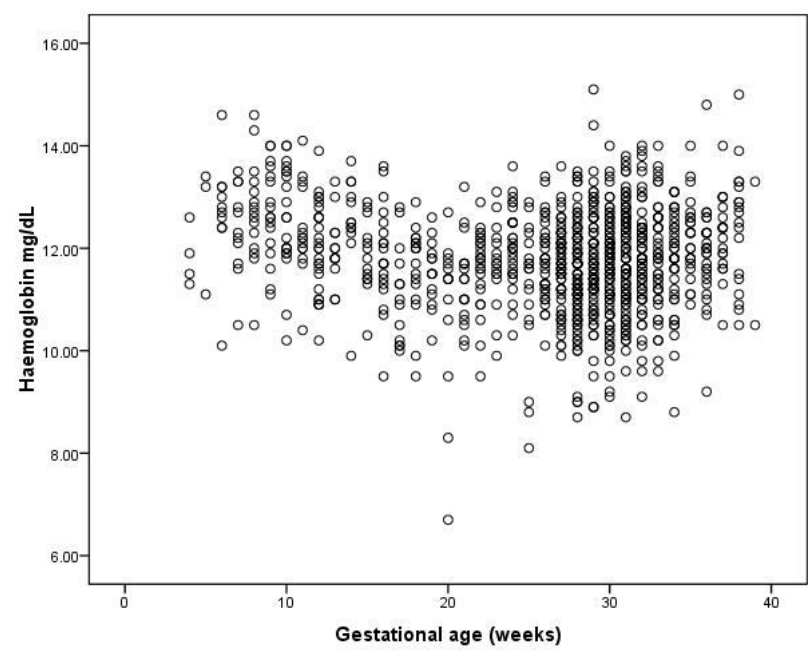

Figure 2. Distribution of serum Hb values by gestational age.

\section{Discussion}

The present study shows that the gestational age adjusted anaemia prevalence in Anuradhapura district as $14.1 \%$ (95\% CI 12.0-16.4\%). Prevalence of severe anaemia was only $0.1 \%$ and, $3.4 \%$ of the study population had moderate anaemia. None of the evaluated sociodemographic factors were associated with $\mathrm{Hb}$ levels except the BMI at booking visit (measured prior to 12 weeks of gestation). Present screening methods showed low sensitivity in detecting anaemia in pregnancy.

Sri Lanka has recently moved up to lower middle income country category from least income country category. This economic growth with improvement of other social determinants, comprehensive and widely appreciated maternal health programme, primary health care system with island wide coverage and multiple evidence based interventions to reduce anaemia in pregnancy would have had a great impact on maternal health improvement in Sri Lanka. However, this was not observed with regard to anaemia during pregnancy, at least according to national and WHO estimates which showed more than $30 \%$ prevalence. In the present community based study, we showed that these national and WHO figure is a gross overestimation of the actual prevalence as shown by other investigators in different parts of the country. Previous studies conducted in Ragama and Colombo where lower prevalence were reported, one explanation could have been the higher socio-economic conditions in those districts. But, Anuradhapura is one of the poorest districts in Sri Lanka. Yet, our estimates are less than half of national and WHO estimates.

Iron deficiency is considered as the leading cause of anaemia in Sri Lanka. A study conducted in Galle among adolescent females (12-16 years) reported a $40 \%$ prevalence of iron deficiency anaemia in 2006 [12]. Around $69 \%$ of pregnant women attending antenatal clinics in 
Colombo were having iron deficiency and the $\mathrm{Hb}$ level was shown as a poor indicator of iron deficiency [13]. The present study is limited due to lack of serum iron studies. However, low prevalence of microcytic hypochromic anaemia raises the question that iron deficiency may not be the leading cause of anaemia in this study population. However, it needs further investigation, as the present study did not assess the iron status directly.

This study shows number of areas to be improved in the present screening programme. As proven repeatedly in other studies, conjunctival method is a poor screening test in detecting anaemia [14]. HCS was developed by WHO as an inexpensive and simple method of haemoglobin assessment for resource poor settings [15]. In the validation study it shows a higher sensitivity of $89 \%$ [16]. Sensitivity was shown to as high as $92 \%$ for severe anaemia [17]. A systematic evaluation on HCS method concludes that "HCS may improve anaemia diagnosis where there is no laboratory, but there is a need for policy-relevant diagnostic research which is pragmatic, implementation-focused and assesses clinical outcomes" [18]. However, few independent studies done later on this have shown very poor sensitivity, as low as $30 \%$ [19]. Low validity of HCS observed in our study could be due to a result of low reliability as observed earlier in field settings of Sri Lanka [10] or may be due to an actual validity issue of the test as shown in another recent study conducted in Colombo [20].

Second issue of screening, is the timing of screening for anaemia. The physiological process of pregnancy clearly shows haemodilution in the second and third trimesters which in turn shows as higher levels of anaemia, which we observed in this study as well. Some authors even suggest that the cut off values for anaemia should be lower in second and third trimesters. Nevertheless, WHO Expert Committee on Anaemia recommends a cut off level of $11 \mathrm{~g} / \mathrm{dl}$, according to which prevalence of anaemia in the second and third trimesters was nearly three times higher than the first trimester. The present guidelines in Sri Lankan pregnancy care programme recommends screening for anaemia in the booking visit and in the third trimester. However, due to resource limitations, screening is carried out only in the booking visit (mostly using HCS). Even in areas where serum $\mathrm{Hb}$ level is tested, the practice is to assess $\mathrm{Hb}$ level at booking visit and repeat only if necessary. These subsequent requests usually depend on clinical examinations, which is insensitive as shown in this study as well as in previous studies. These issues systematically overlook anaemic pregnant women in second and third trimesters and it might have a profound effect on maternal and newborn health.

This study shows the inaccuracies in applying national estimations of anaemia in pregnancy in subnational levels and need for updating and evaluating the area specific situations in order to plan local preventive programmes. Further we showed probable under diagnosis of anaemic pregnant women due to problems of methods and timing of anaemia screening in Sri Lankan maternal health programme. An evaluation of present screening methods and providing recourses for serum $\mathrm{Hb}$ testing, rather than HCS screening in areas where those facilities are not available is strongly recommended.

\section{Acknowledgments}

This study was funded by the Maternal Health Task Force of EngenderHealth.

\section{References}

1. Piyasena C, Mahamithawa AMASB. Assessment of anaemia status in Sri Lanka 2001-2003, Medical Research Institute, Ministry of Health, Nutrition and Welfare, Department of Health Services Colombo, 2003.

2. Department of Census and Statistics Sri Lanka, Prevalence of Anaemia among Children and Women, Demographic and Health Survey 2006/7, Health Sector Development Project, Ministry of Healthcare and Nutrition: Colombo, 2009, 27.

3. Fernandopulle PS. Prevalence of anaemia and some risk factors in pregnant women in DDHS area Dankotuwa, in Community Medicine. Postgraduate Institute of Medicine, University of Colombo, 1999.

4. Abeysena C, Jayawardana P, de A Senevirathna R. Maternal haemoglobin level at booking visit and its effect on adverse pregnancy outcome. Australian and New Zealand Journal of Obstetrics and Gynaecology 2010; 50: 423-7.

5. Sivaganesh S, Senarath U. Prevalence of antenatal risk conditions among women in an underserved district of Northern Sri Lanka. Ceylon Medical Journal 2009; 54: $110-4$.

6. Department of Census and Statistics Sri Lanka. Estimated Mid Year Population. 2011 [cited 2011 October 13]; Available from: http://www.statistics.gov.lk/PopHouSat/Mid\%20 Year\%20Population/midyearsex\&district.pdf.

7. Summary of Quarterly Maternal and Child Health Report (H509) Anuradhapura district, MOMCH Anuradhapura, 2009.

8. Henderson RH, Sundaresan T. Cluster sampling to assess immunization coverage: a review of experience with a simplified sampling method. Bulletin of World Health Organization 1982; 60: 253-60.

9. Dahanayaka NJ, Agampodi SB, Ranasinghe ORJC, et al. Inadequacy of the risk factor based approach to detect gestational diabetes mellitus. Ceylon Medical Journal 2011; 57: 5-9

10. Agampodi S, Kularathna S, Pathiraja I, Intra-rater and Interrater reliability of hemoglobin color scale method. Indian Journal of Community Medicine 2009; 34: 367-8.

11. Benoist B, McLean E, Cogswell M, et al, Worldwide prevalence of anaemia 1993-2005: WHO global database on anaemia. World Health Organization: Geneva. 40, 2008.

12. Hettiarachchi M, Liyanage $\mathrm{C}$, Wickremasinghe $\mathrm{R}$, et al. Prevalence and severity of micronutrient deficiency: a crosssectional study among adolescents in Sri Lanka. Asia Pacific Journal of Clinical Nutrition 2006; 15: 56-63. 
13. Goonewardene M, Seekkuge J, Liyanage C. Iron stores and its correlation to haemoglobin levels in pregnant women attending an antenatal clinic. Ceylon Medical Journal 1995; 40: 67-9.

14. Chalco JP, Huicho L, Alamo C, et al. Accuracy of clinical pallor in the diagnosis of anaemia in children: a meta-analysis. BMC Pediatrics 2005; 5: 46.

15. Stott GJ, Lewis SM. A simple and reliable method for estimating haemoglobin. Bulletin of World Health Organization 1995; 73: 369-73.

16. Münster M, Lewis SM, Erasmus LK, Mendelow BV. Field evaluation of a novel haemoglobin measuring device designed for use in rural setting. South African Medical Journal 1997; 87: 1522-6.
17. Montresor A, Ramsan M, Khalfan N, et al. Performance of the haemoglobin colour scale in diagnosing severe and very severe anaemia. Tropical Medicine and International Health 2003; 8: 619-24.

18. Critchley J, Bates I. Haemoglobin colour scale for anaemia diagnosis where there is no laboratory: a systematic review. International Journal of Epidemiology 2005; 34: 1425-34.

19. Anand H, Mir R, Saxena R. Haemoglobin color scale a diagnostic dilemma. Indian Journal of Pathology and Microbiology 2009; 52: 360-2.

20. Prathapan S, Lindmark G, Fonseka $\mathrm{P}$, et al. How good is the quality of antenatal care in the Colombo district of Sri Lanka in diagnosing and treating anaemia? Quality in Primary Care 2011; 19: 245-50. 